\title{
Construcción de una Escala para Evaluar Emociones de Logro en Estudiantes de Primaria
}

\section{Construction of a Scale to Assess Achievement Emotions in Elementary Students}

\author{
Thamir Durán Fonseca ${ }^{1}$ y Guadalupe Acle-Tomasini ${ }^{2}$
}

\begin{abstract}
Resumen
Las emociones vivenciadas por los alumnos en entornos educativos promueven o limitan su interés por el estudio (Kim \& Hodges, 2012), por lo que es importante elaborar instrumentos en español para su evaluación en estudiantes. Por ello, el objetivo del estudio fue construir una Escala de Emociones de Logro para Estudiantes de Primaria. La muestra se conformó por 527 estudiantes de cuarto a sexto grado de escuelas públicas. Inicialmente se elaboraron diversos reactivos con base en un estudio exploratorio y los supuestos teóricos. Posteriormente, los ítems fueron analizados con razón e índice de validez de contenido, correlaciones, consistencia interna y análisis factoriales. Los resultados mostraron una escala de 10 reactivos con dos factores: (a) emociones positivas de activación durante la actividad y (b) emociones retrospectivas de resultado. Se obtuvieron evidencia de confiabilidad y validez de contenido, constructo y criterio.
\end{abstract}

Palavras-chave: educación básica, instrumento de medida, emoción

\begin{abstract}
Emotions experienced by students in educational environments promote or limit their interest in studying (Kim \& Hodges, 2012), so it is important to develop instruments in Spanish for assessment in students. Therefore, the aim of the study was to construct an Achievement Emotions Scale for Elementary School Students. The sample consisted of 527 fourth to sixth grade public school students. Initially, various items were developed based on an exploratory study and theoretical assumptions. Later, the items were analyzed with reason and content validity index, correlations, internal consistency and factor analysis. The results showed a scale of 10 items with two factors: (a) positive activation emotions during activity and (b) retrospective outcome emotions. Evidence of reliability and validity of content, construct and criteria was obtained.
\end{abstract}

Keywords: basic education, measuring instrument, emotion

\footnotetext{
${ }^{1}$ Doctor en Psicología. Profesor Ordinario de Asignatura de Posgrado. Universidad Nacional Autónoma de México. Batalla 5 de mayo S/N, esquina fuerte de Loreto, Colonia Ejército de Oriente, Iztapalapa, C.P. 09230, Ciudad de México, México. Tel.: 5951336264. Correo: dandu22@hotmail.com

${ }^{2}$ Licenciada en Psicología y Doctora en Antropología Social. Docente y Tutora del Programa de Posgrado en Psicología. Universidad Nacional Autónoma de México. Batalla 5 de mayo S/N, esquina fuerte de Loreto, Colonia Ejército de Oriente, Iztapalapa, C.P. 09230, Ciudad de México, México. Tel.: 5565182230. Correo: gaclet@unam.mx 


\section{Introducción}

El estudio de las emociones ha sido relevante en la investigación psicológica desde hace varios años, sin embargo, recientemente han incursionado en el campo de la educación como una variable de interés para el entendimiento de la enseñanza y el aprendizaje. De acuerdo con Pintrich y Schunk (2006), la importancia de considerarlas dentro del contexto académico, se debe a que generan influjos importantes en las decisiones que toman los estudiantes, además de que promueven o limitan el interés de los alumnos en las actividades escolares (Kim \& Hodges, 2012). No obstante, tales supuestos no han sido suficientes para incitar la construcción de instrumentos con evidencias de confiabilidad y validez para su evaluación en estudiantes hispanohablantes, a pesar de que en esta etapa se presentan las bases del aprendizaje.

Los aportes que consideraron las emociones de logro por primera vez, fueron expuestos en la teoría del control-valor desarrollada por Pekrun et al. (2007). Las concepciones sobre el controlvalor complementaron las teorías de la atribución causal de Weiner $(1972,1985,2010)$ y la expectativa-valor de Wigfield y Eccles (1992, 2000), al considerar que las emociones experimentadas durante el proceso educativo, son generadas al margen de cualquier atribución causal, y que son parte del valor subjetivo que los estudiantes adjudican a la actividad (Pekrun et al., 2007).

Desde esta perspectiva, para Pekrun et al. (2007) el valor que se asigna a las actividades no depende sólo del logro, el interés, la utilidad y el costo, tal como lo exponen Wigfield y Eccles (1992, 2000), sino que, también se estructura por las emociones. Por ejemplo, las actividades que aburren o enojan a los estudiantes tienden a ser menos valoradas por ellos. También, la teoría del control-valor integra otras emociones, y no sólo considera el disfrute, a diferencia del enfoque de expectativa-valor (Wigfield \& Eccles, 2000).

Para Frenzel et al. (2007), Pekrun et al. (2011) y Pekrun et al. (2002), los alumnos vivencian una gran variedad de emociones en el contexto escolar. Entre éstas destacan el disfrute, enojo, aburrimiento, desesperación y ansiedad como las emociones que experimentan los alumnos con mayor frecuencia (Frenzel et al., 2007). Otro aspecto característico de la teoría del controlvalor, es que sólo se consideran las emociones que están directamente asociadas con las actividades escolares y los resultados (Frenzel et al., 2007; Pekrun et al., 2007; Pekrun et al., 2011). Tal como, el disfrute, la desesperación y el aburrimiento durante la resolución de operaciones matemáticas, o la ansiedad, enojo, tristeza y alegría al conocer la calificación obtenida en el examen.

Por ello, Frenzel et al. (2007) definieron las emociones de logro como las emociones que los estudiantes experimentan en entornos educativos, durante la ejecución de las actividades y debido a los resultados obtenidos. Además, para Pekrun et al. (2007) existen dos tipos de emociones de resultado, las prospectivas, que se dan al anticipar el éxito o fracaso en el resultado, y las retrospectivas, que se experimentan después del resultado. Estas últimas se relacionan con los supuestos de Weiner $(1972,1985,2010)$.

Otra forma de clasificar las emociones, es por medio de los criterios de valencia y activación fisiológica (Kleine et al., 2005; Pekrun et al., 2009; y Pekrun et al., 2011). La valencia se refiere a la tipología clásica de positivo-negativo para describir emociones agradables y desagradables. Por su parte, la activación fisiológica se basa en la concepción de activación e inhibición. Por lo tanto, desde este punto de vista, se pueden identificar cuatro grupos de emociones: (a) positivas de activación, en las que se incluyen disfrute, alegría, esperanza, orgullo y gratitud; (b) positivas de inhibición, en las que se consideran la tranquilidad-relajación y el alivio; (c) negativas de activación, entre las que se encuentran enojo, frustración, ansiedad, desesperación y vergüenzaculpa; y (d) negativas de inhibición con las emociones de aburrimiento, tristeza, decepción y desesperanza.

Sin embargo, para Pekrun et al. (2002) la categorización de emociones negativas como perjudiciales y positivas como benéficas, resulta errónea en este campo de análisis. Ambas pueden promover o inhibir la motivación de los alumnos, ya que las emociones negativas ejercen una influencia ambivalente. En representación, el enojo, la vergüenza y la ansiedad, pueden fortalecer los esfuerzos para evitar el fracaso. En 
Tabla 1. Características de los instrumentos que evalúan emociones de logro

\begin{tabular}{|c|c|c|c|c|}
\hline Instrumento & Tipo de medición & Dimensiones & Población & Características psicométricas \\
\hline $\begin{array}{l}\text { Achievement } \\
\text { Emotions } \\
\text { Questionnaire } \\
\text { (Pekrun et al., } \\
\text { 2011). }\end{array}$ & $\begin{array}{l}232 \text { reactivos tipo } \\
\text { Likert de cinco } \\
\text { puntos. }\end{array}$ & $\begin{array}{c}24 \text { factores que miden } \\
\text { disfrute, esperanza, orgullo, } \\
\text { enojo, ansiedad, vergüenza, } \\
\text { desesperanza y aburrimiento, } \\
\text { durante la clase, mientras se } \\
\text { estudia y al presentar } \\
\text { exámenes. }\end{array}$ & $\begin{array}{c}389 \\
\text { universitarios } \\
\text { canadienses } \\
(M=20.63 \\
\text { años })\end{array}$ & $\begin{array}{c}\text { Evidencia de confiabilidad en todos } \\
\text { los factores }(\alpha>.75) \text {. Evidencias de } \\
\text { validez de constructo y criterio. AFC } \\
{\left[\chi^{2}(134)=370.78 \text {; GFI }=.92, \mathrm{CFI}=.99, \mathrm{y}\right.} \\
\mathrm{RMSEA}=.072] . \text { Correlaciones }(p<.05) \\
\text { de las emociones con autoeficacia } \\
(.27<r<.60) \text {, valor a la tarea } \\
(.13<r<.55), \text { motivación }(.10<r<.49) \text { y } \\
\text { rendimiento }(.14<r<.34) .\end{array}$ \\
\hline $\begin{array}{l}\text { Achievement } \\
\text { Emotions } \\
\text { Questionnaire- } \\
\text { Elementary } \\
\text { School (AEQ- } \\
\text { ES) (Lichtenfeld } \\
\text { et al., 2012). }\end{array}$ & $\begin{array}{l}28 \text { reactivos } \\
\text { pictográficos tipo } \\
\text { Likert de cinco } \\
\text { puntos. }\end{array}$ & $\begin{array}{l}\text { Ocho factores que consideran } \\
\text { el disfrute, la ansiedad y el } \\
\text { aburrimiento experimentados } \\
\text { durante la clase, al hacer la } \\
\text { tarea y resolver exámenes. } \\
\text { Enfocado en las matemáticas. }\end{array}$ & $\begin{array}{c}1189 \\
\text { estudiantes de } \\
\text { primaria } \\
(M=9.05 \text { años }) \\
\text { en Alemania y } \\
\text { EU. }\end{array}$ & $\begin{array}{c}\text { Evidencia de confiabilidad en todos } \\
\text { los factores }(.71<\alpha<.93) \text {. Evidencias } \\
\text { de validez de constructo y criterio. } \\
\text { AFC }\left(\chi^{2}=1223.49, p<.01 ; \text { SRMR }=.05 \text {, }\right. \\
\text { CFI }=.92 \text {, TLI }=.91 \text { y RMSEA }=.066) . \\
\text { Correlaciones }(p<.05) \text { de la escala con } \\
\text { valor a la tarea }(.26<r<.54) \text { y } \\
\text { rendimiento en matemáticas } \\
(.10<r<.37) .\end{array}$ \\
\hline $\begin{array}{l}\text { Achievement } \\
\text { Emotions } \\
\text { Questionnaire. } \\
\text { Revision de } \\
\text { Paoloni et al. } \\
\text { (2014). }\end{array}$ & $\begin{array}{l}80 \text { reactivos tipo } \\
\text { Likert de cinco } \\
\text { puntos. }\end{array}$ & $\begin{array}{c}\text { Ocho factores sobre } \\
\text { emociones experimentadas } \\
\text { en clase; disfrute, esperanza, } \\
\text { orgullo, enojo, ansiedad, } \\
\text { vergüenza, desesperanza y } \\
\text { aburrimiento. }\end{array}$ & $\begin{array}{l}450 \\
\text { universitarios } \\
\text { argentinos de } \\
18 \text { a } 21 \text { años. }\end{array}$ & $\begin{array}{l}\text { Evidencia de confiabilidad total del } \\
\text { instrumento }(\alpha=.88) \text {. Evidencia de } \\
\text { validez de contenido con } 80 \% \text { de } \\
\text { acuerdo entre jueces. }\end{array}$ \\
\hline $\begin{array}{l}\text { Achievement } \\
\text { Emotions } \\
\text { Questionnaire- } \\
\text { Argentine (AEQ- } \\
\text { AR) (Sánchez, } \\
\text { 2015). }\end{array}$ & $\begin{array}{l}206 \text { reactivos tipo } \\
\text { Likert de cinco } \\
\text { puntos. }\end{array}$ & $\begin{array}{l}24 \text { factores que miden } \\
\text { disfrute, esperanza, orgullo, } \\
\text { enojo, ansiedad, vergüenza, } \\
\text { desesperanza y aburrimiento, } \\
\text { en clase, al estudiar y } \\
\text { resolver exámenes. }\end{array}$ & $\begin{array}{c}666 \\
\text { universitarios } \\
\text { argentinos } \\
(M=25.09 \\
\text { años })\end{array}$ & $\begin{array}{c}\text { Evidencia de confiabilidad total del } \\
\text { cuestionario }(\alpha=.90) \text {. Evidencias de } \\
\text { validez de constructo y criterio. } \\
\text { Análisis Factorial Exploratorio (AFE) } \\
\text { [KMO }=.89 \text { y } 59 \% \text { de varianza } \\
\text { explicada]. AFC }\left(\chi^{2} / g l<2.94 \text {; CFI } \geq .98 \text {, }\right. \\
\text { GFI } \geq .97 \text { y RMSEA } \leq .08) . \\
\text { Correlaciones }(p<.05) \text { con autoeficacia } \\
(.13<r<.68) \text {, valor a la tarea } \\
(.10<r<.62) \text { y rendimiento } \\
(.08<r<.32) .\end{array}$ \\
\hline $\begin{array}{l}\text { Achievement } \\
\text { Emotions } \\
\text { Questionnaire } \\
\text { for Pre- } \\
\text { Adolescents } \\
\text { (AEQ-PA) } \\
\text { (Peixoto et al., } \\
\text { 2015). }\end{array}$ & $\begin{array}{l}48 \text { reactivos tipo } \\
\text { Likert de cinco } \\
\text { puntos. Se } \\
\text { consideraron dos } \\
\text { escalas con } 24 \\
\text { ítems cada una. 1) } \\
\text { Emociones en } \\
\text { clase y, 2) } \\
\text { Emociones en } \\
\text { exámenes. }\end{array}$ & $\begin{array}{c}\text { Cada escala se compone de } \\
\text { seis factores. Están enfocadas } \\
\text { en las matemáticas y } \\
\text { consideran; aburrimiento, } \\
\text { desesperanza, enojo, } \\
\text { ansiedad, disfrute, orgullo, } \\
\text { esperanza, vergüenza y } \\
\text { alivio. }\end{array}$ & $\begin{array}{l}1515 \\
\text { estudiantes } \\
\text { portugueses } \\
(M=11.13 \\
\text { años })\end{array}$ & $\begin{array}{c}\text { Evidencia de confiabilidad de todos } \\
\text { los factores }(\alpha \geq .75) \text {. Evidencias de } \\
\text { validez de constructo. AFC } \\
\left(\chi^{2} / g l \leq 5.24 ; \text { CFI } \geq .94, \text { TLI } \geq .93 \text { y }\right. \\
\text { RMSEA } \leq .053) .\end{array}$ \\
\hline
\end{tabular}

este sentido, algunos estudios han reportado que las emociones académicas están significativamente relacionadas con la motivación escolar (Kim \& Hodges, 2012; Kleine et al., 2005; Pekrun et al., 2009; Pekrun et al., 2011).

En la investigación desarrollada en Alemania por Pekrun et al. (2002) con estudiantes universitarios, se encontró que las emociones de disfrute, esperanza, orgullo, aburrimiento $\mathrm{y}$ desesperanza, están significativamente relacionadas con la motivación. Sus datos también demostraron que la ansiedad fue la emoción más reportada. De manera más detallada, los datos de Pekrun et al. (2011) con universitarios canadienses, indicaron que las emociones positivas de activación (disfrute, esperanza y orgullo), se relacionan positivamente con la valoración de la actividad y la motivación.

Otros resultados han evidenciado la asociación de las emociones de logro con el rendimiento académico (Herrera, 2014; Valle et al., 2015; Viljaranta, 2010), incluso a nivel predictivo (Pekrun, et al., 2009). Por ejemplo, Kleine et al. (2005) encontraron que los alumnos alemanes de quinto grado de primaria y con alto desempeño, se caracterizaron en sentir altos niveles de disfrute. 
En términos de los instrumentos para medir este tipo de emociones, el trabajo de Pekrun et al. (2004) fue pionero con la creación del Test Emotions Questionnaire (TEQ). Antes de éste, las escalas sólo evaluaban la ansiedad asociada con los exámenes, aunque actualmente existen otras pruebas, como la desarrollada por CastilloNavarrete et al. (2020) que consideran otras dimensiones, como el estrés.

Sin embargo, además de la ansiedad, el TEQ integra disfrute, esperanza, orgullo, alivio, enojo, vergüenza y desesperanza. Con base en estudios cualitativos, el trabajo de estos autores se desarrolló con estudiantes universitarios de Alemania y Canadá, con los que obtuvieron evidencias de validez de criterio, constructo y confiabilidad $(.88<\alpha<.95)$. Sus resultados fueron correlacionados con diversas variables, entre las que se encontraban la autoeficacia y el interés, éste último como elemento motivacional. En todos los casos encontraron correlaciones significativas $(.10<r<.60 ; p<.05)$. Para la validez de constructo realizaron diversos Análisis Factoriales Confirmatorios (AFC). En algunos casos se identificaron índices mínimamente aceptables $\quad\left(\chi^{2} / \mathrm{gl}=4.30 ; \quad \mathrm{GFI}=.91, \quad \mathrm{CFI}=.92\right.$ y RMSEA $=.09)$ y en otras ocasiones, valores óptimos $\quad\left(\chi^{2} / \mathrm{gl}=1.67 ; \quad \mathrm{GFI}=.98, \quad \mathrm{CFI}=.98 \quad \mathrm{y}\right.$ RMSEA=.04). A partir de este instrumento, han surgido otros similares, como se muestra en la Tabla 1.

Cabe resaltar que los instrumentos encontrados poseen evidencias sólidas de confiabilidad y validez de constructo, pero carecen de al menos un tipo de validez, la mayoría de contenido, y han sido elaborados para estudiantes universitarios y/o en países con idiomas diferentes al español.

Algunos autores consideran que son escasos los estudios instrumentales que evalúan algún aspecto afectivo en población infantil (Arrivillaga \& Extremera, 2020; Jiménez-Blanco et al., 2020) y de Latinoamérica (Castillo-Navarrete et al., 2020). Las altas cargas de trabajo, el estrés culminante durante los estudios superiores (Castillo-Navarrete et al., 2020) y las dificultades teórico metodológicas en la evaluación en niños (Jiménez-Blanco et al., 2020), probablemente promueven el desarrollo prioritario de instrumentos en adolescentes y adultos. Además, para Arrivillaga y Extremera (2020) es común que en las investigaciones se utilicen escalas provenientes de otros países, sin analizar la pertinencia de los significados culturales, situación que afecta la calidad de los hallazgos.

En consecuencia, no se encontraron instrumentos con evidencias sobre la validez global y ninguno de ellos evalúa las emociones de logro en estudiantes de primaria de habla hispana, a pesar de ser una población relevante por su etapa en el desarrollo. De ahí el interés por diseñar una escala qué permita evaluar las emociones de logro, y que la información obtenida en ésta permita realizar investigaciones futuras y la instrumentación de programas, intervenciones y talleres que impacten en el desempeño académico de los estudiantes. Por ello, el objetivo del estudio fue construir una Escala de Emociones de Logro para Estudiantes de Primaria (EELEP). Para lograrlo se plantearon los siguientes objetivos específicos: (a) identificar los significados de actividades académicas de aprendizaje y emociones de logro; (b) obtener evidencias de validez de contenido, criterio y constructo; y (c) obtener evidencia de confiabilidad.

\section{Método}

\section{Participantes}

Se seleccionaron por un muestreo no probabilístico por cuotas 527 estudiantes de $4^{\text {to }}$ a $6^{\text {to }}$ grado de primaria con el propósito de tener proporciones similares y suficientes para cada grado escolar (Kerlinger \& Lee, 2002). El 32.15\% estudiaban el cuarto grado, $33.1 \%$ el quinto y $34.75 \%$ el sexto. Su edad osciló entre los 8 y los 13 años $(M=10.03 ; D E=0.92)$ y el $55.05 \%$ fueron niñas. Todos pertenecían a escuelas públicas de la Zona Metropolitana del Valle de México. Del total de la muestra, 15 estudiantes participaron en la fase inicial para cumplir con el primer objetivo específico, otros 14 fueron elegidos para realizar el piloteo de la prueba, 250 para una primera aplicación de la EELEP y los últimos 248 para una segunda aplicación de la EELEP, además de otros instrumentos utilizados. Las dos últimas sub-muestras de estudiantes fueron utilizadas para evaluar las características psicométricas de confiabilidad y validez. 
Tabla 2. Proporción de estudiantes por sexo y grado

\begin{tabular}{llccccc}
\hline & \multicolumn{4}{c}{ Proporción de estudiantes } \\
\cline { 3 - 6 } & & \multicolumn{2}{c}{ Sexo } & Crado \\
\cline { 3 - 6 } & Niños & Niñas & Cuarto & Quinto & Sexto \\
\hline \multirow{2}{*}{ Fase del } & Inicial & $8(53.33 \%)$ & $7(46.66 \%)$ & $5(33.33 \%)$ & $6(40 \%)$ & $4(26.66 \%)$ \\
estudio & Piloteo & $9(64.29 \%)$ & $5(35.71 \%)$ & $5(35.71 \%)$ & $4(28.58 \%)$ & $5(35.71 \%)$ \\
& Primera aplicación & $117(46.80 \%)$ & $133(53.20 \%)$ & $74(29.60 \%)$ & $93(37.20 \%)$ & $83(33.20 \%)$ \\
& Segunda aplicación & $107(43.14 \%)$ & $141(56.85 \%)$ & $86(34.67 \%)$ & $72(29.03 \%)$ & $90(36.29 \%)$ \\
\hline
\end{tabular}

Los grupos compartían características similares en cuanto a la proporción por sexo y grado. De acuerdo con los resultados de la prueba Chi cuadrado, no se encontraron diferencias significativas entre las frecuencias de niños y niñas $\left[\chi^{2}(3)=3.07, p=.38\right]$, ni entre los grados escolares $\left[\chi^{2}(6)=4.48, p=.612\right]$. En la Tabla 2 se muestran las proporciones por sexo y grado de los estudiantes.

\section{Instrumentos}

Guión para grupo focal (Rea \& Acle, 2017). Se estructura de seis fases que describen los pasos para llevar a cabo un grupo focal: (1) apertura, que incluye la bienvenida, el establecimiento de reglas, la explicación de los objetivos y el papel del moderador, así como la presentación de los participantes; (2) calentamiento, la cual tiene el propósito de realizar preguntas introductorias para inducir a los participantes en la temática; (3) clarificación de términos, en la que se establecen los conocimientos básicos de los términos clave; (4) fase central, que genera una relación activa entre el moderador y los participantes, para que estos últimos respondan las preguntas; (5) cierre, que tiene los propósitos de clarificar la información obtenida sobre los temas tratados y de llegar a un consenso en las conclusiones finales; y (6) declaración de cierre, en la cual se responden dudas y se agradece la participación.

Guía de entrevista semi-estructurada sobre emociones de logro. Instrumento creado para el presente estudio que se compone de dos apartados. En un principio se solicitan los datos sociodemográficos de los integrantes del grupo, tales como; nombre de la escuela, grupo, turno, jornada escolar, sexo y edad. Posteriormente se encuentran siete preguntas abiertas, tres sobre las materias escolares que median su aprendizaje y cuatro sobre las emociones que vivencian los estudiantes en la escuela.

Las preguntas sobre las materias fueron las siguientes; ¿Saben cuáles son las actividades escolares que les ayudan a aprender?, ¿Cómo les llamarían a esas actividades? y ¿Qué otras actividades se les ocurren? En el caso de las emociones de logro se preguntó; ¿Qué significa cuando un niño dice que tiene emociones al hacer las actividades (trabajos) escolares?, ¿Cuáles emociones sienten los alumnos cuando hacen actividades escolares?, ¿Cuáles emociones sienten los alumnos sobre sus calificaciones? Y ¿Cómo son esas emociones? Se consideró importante incluir un apartado sobre las materias para identificar las actividades que los estudiantes consideraban relacionadas con su aprendizaje, ya que algunas de las actividades que realizan en la escuela no están directamente vinculadas con su aprendizaje escolar.

Escala de Motivación Escolar para Alumnos de Primaria (Durán \& Acle, 2021). Cuenta con 16 reactivos, cinco opciones de respuesta pictográficas que van de nunca a siempre y tres factores; persistencia, esfuerzo y disposición por aprobar exámenes. De acuerdo con los autores, posee evidencias de validez de contenido [Razón de Validez de Contenido (RVC) e Índice de Validez de Contenido (IVC) superiores a .60], criterio ( $r=.23, p=.001$ con el promedio de calificaciones) y constructo, este último a través de análisis factorial exploratorio (varianza explicada de $55 \% ; \mathrm{KMO}=.92 ; \chi^{2}[(102)=173.5$, $p<.001])$ y confirmatorio $\left(\chi^{2} / g l=2.07 ; \mathrm{CFI}=.91\right.$, $\mathrm{IFI}=.92, \mathrm{GFI}=.91$ y RMSEA=.07). Con la muestra del presente estudio, se encontró un alfa de Cronbach para los factores de; .81, .79 у .71, respectivamente.

Escala de Valor a la Tarea en Alumnos de Primaria (Durán \& Acle, 2019). Se conforma por 17 reactivos, cinco opciones de respuesta pictográficas que van de nunca a siempre y cinco factores; valía hacia las matemáticas, utilidad, valía hacia la geografía, costo y valía hacia la historia. Con base en los autores, cuenta con evidencias de validez de contenido [Razón de 
Validez de Contenido (RVC) e Índice de Validez de Contenido (IVC) superiores a .60], criterio ( $r=.62, \quad p<.001 \quad$ con motivación escolar) $\mathrm{y}$ constructo, este último a través de análisis factorial exploratorio (varianza explicada de $\left.44.52 \% ; \mathrm{KMO}=.84 ; \chi^{2}[(340)=397.21, p=.02]\right) \mathrm{y}$ confirmatorio $\quad\left(\chi^{2} / g l=2.6 ; \quad \mathrm{CFI}=.85, \quad \mathrm{IFI}=.86\right.$, $\mathrm{GFI}=.90$ y RMSEA=.08). La consistencia interna de los factores y con la muestra de esta investigación fue de; .73, .75, .60, 62 y .50 de manera respectiva.

Escala de Autoconcepto Académico para Alumnos de Primaria (Durán \& Acle, 2020). Se estructura de 12 reactivos, cinco opciones de respuesta pictográficas que van de nunca a siempre y dos factores; autovaloración positiva y automonitoreo. Los autores indicaron evidencias de validez de contenido [Razón de Validez de Contenido (RVC) e Índice de Validez de Contenido (IVC) superiores a .60], criterio ( $r=.68$, $p<.01$ con motivación escolar) y constructo, este último a través de análisis factorial exploratorio (varianza explicada de 38\%; $\mathrm{KMO}=.90 ; \chi^{2}$ $[(134)=205.63, \quad p<.001])$ y confirmatorio $\left(\chi^{2} / g l=1.67 ; \quad\right.$ CFI $=.95, \quad$ IFI $=.95, \quad$ GFI $=.94 \quad \mathrm{y}$ RMSEA=.05). Con los participantes de este estudio, los valores alfa de Cronbach fueron de; .78 para el primer factor y .70 para el segundo.

\section{Procedimiento}

La investigación se clasificó dentro del enfoque empírico cuantitativo de tipo instrumental. De acuerdo con Montero y León (2007) en estos estudios se desarrollan pruebas y se describen las propiedades psicométricas de los instrumentos.

Inicialmente se realizaron tres grupos focales con cinco estudiantes cada uno, para identificar el significado sobre las actividades relevantes para su aprendizaje escolar y las emociones vivenciadas en la escuela. Las respuestas fueron audio-grabadas y transcritas para su análisis. De acuerdo con estos resultados y los supuestos teóricos, se elaboraron los reactivos para medir las emociones de logro. Más tarde, cinco mujeres participaron como jueces expertos $(M=35$ años; $D E=5)$ para evaluar los reactivos de la Escala de Emociones de Logro para Estudiantes de Primaria, en términos de suficiencia, claridad, coherencia y relevancia. Para ello se utilizó la planilla sobre validación de contenido de EscobarPérez y Cuervo-Martínez (2008). El 80\% de las evaluadoras contaban con estudios de maestría y el 20\% de licenciatura. En promedio tenían 3.4 años de experiencia en la construcción de instrumentos, 8.6 años en la evaluación de niños y 6.6 años de trayectoria docente.

Con base en la evaluación de las expertas, se construyó una versión piloto de la escala y ésta fue aplicada a 14 estudiantes de manera individual. En este proceso se solicitó a los menores que realizaran comentarios sobre; la pertinencia y claridad de las instrucciones, la facilidad para responder los ítems y las dudas que pudieran surgir. Con la información obtenida en el piloteo se decidió no realizar cambios al instrumento.

Posteriormente se aplicó la Escala de Emociones de Logro para Estudiantes de Primaria a 250 estudiantes, con el propósito de identificar evidencias de validez de constructo, por medio de un Análisis Factorial Exploratorio (AFE). Esta sub-muestra fue obtenida de manera intencional. Finalmente, se realizó un muestreo no probabilístico para seleccionar 248 estudiantes. Estos respondieron las escalas de motivación escolar, valor a la tarea $\mathrm{y}$ autoconcepto académico, y con los padres de familia se obtuvo el promedio de calificaciones de los estudiantes en el último trimestre, con la intención de mostrar evidencias de validez de criterio. Asimismo, a esta segunda sub-muestra se le aplicó la prueba de emociones de logro, para realizar Análisis Factorial Confirmatorio (AFC). Todas las aplicaciones fueron de manera grupal, dentro del salón de clases y en horario escolar.

\section{Análisis de datos}

La información obtenida en los grupos focales fue examinada a través del análisis de contenido categorial. Además, las categorías fueron establecidas con mutuo acuerdo entre los investigadores. Las evaluaciones de los jueces fueron analizadas con las modificaciones de Razón de Validez de Contenido (RVC) e Índice de Validez de Contenido (IVC) establecidos por Tristán-López (2008).

A continuación, se realizó un AFE con una sub-muestra de 250 estudiantes. El tratamiento estadístico propuesto por Reyes-Lagunes y García y Barragán (2008) fue utilizado como base: (a) 
análisis de frecuencias y medidas de distribución, con el propósito de verificar que los datos hayan sido capturados correctamente (ningún valor fuera de rango), y que todas las opciones de respuesta fueran atractivas y elegidas por los participantes, con frecuencias inferiores al $50 \%$ en cada una de ellas; (b) $t$ de Student para evaluar la capacidad de discriminación de los reactivos por comparación de grupos extremos (percentiles 25 y 75); y, (c) análisis de consistencia interna alfa de Cronbach.

En cuanto a este último, debido a que el coeficiente de confiabilidad depende de la cantidad de reactivos en la escala (Martínez et al., 2014), con base en Argibay (2006) se consideró que valores superiores a .80 son buenos indicadores en el caso de instrumentos con longitudes extensas (mayores de 30 reactivos), pero en el caso de instrumentos con pocos ítems (10 o menos), menores a .70 denotan buena confiabilidad. Igualmente, se calculó la correlación ítem-total corregida, en la cual, valores por encima de .30 se reconocen como indicadores de homogeneidad de los ítems (Santos-Roig \& Pérez-Meléndez, 2014). Lo cual, indica que los reactivos tienen capacidad discriminativa y que contribuyen significativamente a la puntuación total (Salvo \& Castro, 2013).

Además, con base en algunas consideraciones en psicometría, se optó por una extracción de máxima verosimilitud con matriz de correlaciones de Pearson y una rotación oblimin, de acuerdo con los siguientes criterios propuestos por LloretSegura et al. (2014): a) se tiene que comprobar la adecuación de la matriz mediante la prueba $K M O$; b) es adecuado utilizar la matriz de correlaciones de Pearson cuando existen mínimo cinco opciones de respuesta, las distribuciones de los reactivos son aproximadamente normales (asimetría y curtosis en el rango de -1 a 1) y la muestra se compone entre 200 y 300 participantes. Además, otro criterio para Ferrando y Lorenzo-Seva (2014) consiste en que las correlaciones inter-ítem deben tener magnitudes de .50 o menos; c) el método de máxima verosimilitud es apropiado para la estimación de los factores si los ítems tienen distribuciones normales (asimetría y curtosis menores que 2), incluso cuando los reactivos son ordinales; d) el número de factores a retener depende de la cantidad de ítems (mínimo 4), el tamaño de la saturación (mínimo .40) y la interpretabilidad de la solución con base en la teoría; y, e) se recomienda la rotación oblicua aunque los factores parezcan independientes, entre los destaca oblimin directo.

Posteriormente, se calculó un AFC con método de mínimos cuadrados no ponderados en la sub-muestra de 248 estudiantes. Para el AFC se tomaron en cuenta los aspectos propuestos por Fernández (2008): (a) especificación del modelo por medio de un diagrama de relaciones; (b) identificación del modelo; (c) estimación de los parámetros del modelo; (d) evaluación del ajuste del modelo. Respecto con los indicadores de ajuste, de acuerdo con la literatura en psicometría sobre el AFC, se consideraron aceptables los siguientes valores: $X^{2}$ normalizado entre 1 y 3; GFI superior a .90; AGFI mayor de .80 (Pérez, Chacón \& Moreno, 2000); RMR y RMSEA entre .05 y .08 (Fernández, 2008; Martínez et al., 2014). En función del constructo definido inicialmente, se comparó el modelo propuesto con un modelo unifactorial, tomando en cuenta que los índices comparativos de ajuste IFI y CFI se consideran aceptables en torno a 90 (Fernández, 2008).

Finalmente, se calcularon correlaciones de Pearson de la puntuación total obtenida en la escala de Emociones de Logro, con el promedio de calificaciones y las sumatorias de las puntuaciones en los instrumentos de Motivación Escolar (Durán \& Acle, 2021), Valor a la Tarea (Durán \& Acle, 2019) y Autoconcepto Académico (Durán \& Acle, 2020). El AFC se realizó a través del programa AMOS (v. 24), mientras que el resto de análisis estadísticos fueron calculados con SPSS (v. 24).

\section{Consideraciones éticas}

Se obtuvo el permiso de las autoridades escolares, el consentimiento informado de los padres y el asentimiento de los niños. La participación fue voluntaria y se garantizó que los datos obtenidos serían confidenciales y sólo con fines de análisis. 
Tabla 3. Verbalizaciones de los estudiantes por dimensión y categoría

\begin{tabular}{|c|c|c|}
\hline Dimensión & Categoría & Verbalizaciones \\
\hline \multirow[t]{2}{*}{$\begin{array}{l}\text { Actividades } \\
\text { académicas } \\
\text { de } \\
\text { aprendizaje }\end{array}$} & Asignaturas & $\begin{array}{l}\text { "Las matemáticas son muy importantes, las usas toda la vida". "Por ejemplo, si vamos a una } \\
\text { tienda, ayudamos a la señora con la cuenta". "La más importante es mate y después español". } \\
\text { "En español desarrollamos la lectura y leer nos ayuda a aprender". "Leer es importante para } \\
\text { saber cosas". "También es importante escribir textos". "En cualquier cosa que quieras ser de } \\
\text { adulto tienes que escribir". } \\
\text { "Geografía es importante para aprender sobre el mundo o tu comunidad". } \\
\text { "Historia es importante para aprender sobre el pasado"." "Nos dicen cuando empezaron las } \\
\text { cosas". "Nos ayuda a entender las cosas por su origen". }\end{array}$ \\
\hline & Examen & $\begin{array}{l}\text { "Otra actividad importante es hacer exámenes". "También los exámenes son útiles, para } \\
\text { tener buenas calificaciones y aprender cosas". }\end{array}$ \\
\hline \multirow[b]{2}{*}{$\begin{array}{l}\text { Emociones } \\
\text { de logro }\end{array}$} & $\begin{array}{l}\text { Emociones } \\
\text { durante la } \\
\text { actividad. }\end{array}$ & $\begin{array}{l}\text { "Te sientes bien cuando te gustan los estudios". "Tienes la emoción de alegría cuando haces } \\
\text { las actividades". "Los niños se divierten haciendo esas actividades". "Si le divierte, tiene la } \\
\text { emoción de felicidad". }\end{array}$ \\
\hline & $\begin{array}{l}\text { Emociones de } \\
\text { resultado }\end{array}$ & $\begin{array}{l}\text { "Sentir emociones es estar feliz por tus calificaciones". "Cuando lo hago bien, estoy feliz y } \\
\text { emocionado". "Te da felicidad cuando sacas buenas calificaciones". "Sientes felicidad } \\
\text { cuando sacas } 10 " . \\
\text { "Te sientes triste cuando sacas malas calificaciones". "Sientes tristeza cuando sacas menos } \\
\text { calificaciones, un siete o menos". }\end{array}$ \\
\hline
\end{tabular}

\section{Resultados}

Los resultados se presentan con base a los objetivos y el procedimiento del estudio.

\section{Significados de actividades académicas de aprendizaje y emociones de logro}

En total se identificaron cuatro categorías, dos para la dimensión de actividades académicas de aprendizaje; asignaturas y examen, y el resto sobre las emociones de logro; emociones durante la actividad y emociones de resultado. De acuerdo con los estudiantes, resolver exámenes y las materias de matemáticas, español, historia y geografía, son las actividades escolares que les permiten aprender. A pesar de que en el currículo académico se incluyen otras asignaturas, ellos solamente mencionaron estas cuatro.

En cuanto a las emociones, los estudiantes reportaron sentir afecto positivo cuando estudian en la escuela, tales como gusto, alegría y disfrute. También, se encontró que los estudiantes sienten felicidad y tristeza al recibir sus calificaciones, dependiendo si consideran como alta o baja la nota obtenida o si no lograron el resultado esperado. Cabe destacar que las emociones antes mencionadas fueron referidas de manera unánime en los tres grados escolares. Sólo en el grupo de sexto año mencionaron sentirse aburridos cuando la actividad es repetitiva. En este sentido, reportaron que, existen actividades aburridas, por ejemplo, cuando haces lo mismo una y outra vez, cuando solo coloreas. En la Tabla 3 se muestran las verbalizaciones por categoría.

Además de las emociones reportadas en la Tabla 3, también se identificaron las de temor y enojo, pero de acuerdo con sus verbalizaciones, no están directamente vinculadas con la actividad o el resultado obtenido en el entorno académico. Como muestra, algunos estudiantes mencionaron lo siguiente; Cuando sacas mala calificación y te regañan, te da temor, y Te enojas cuando te regresa el trabajo la maestra. En ambos casos, las emociones surgen por factores externos a la actividad y al resultado. En este sentido, los estudiantes tienen temor por el regaño y no a causa de la calificación, y sienten enojo porque la maestra les regresa el trabajo y no por ejecutar la actividad.

Finalmente, la información recabada sobre los significados, junto con la definición teórica de emociones de logro propuesta por Frenzel et al. (2007), facilitaron la elaboración de 10 reactivos para evaluar la variable, cinco sobre las emociones vivenciadas durante la ejecución de las actividades escolares y cinco sobre las emociones asociadas con los resultados retrospectivos. Por lo tanto, de acuerdo con las verbalizaciones de los estudiantes, la creación de los ítems se basó en las emociones de; gusto, disfrute, felicidad y tristeza, $\mathrm{y}$ en las actividades escolares relacionadas con; matemáticas, geografía, historia, exámenes y la escritura. Los reactivos elaborados y su dimensión teórica correspondiente se muestran en la Tabla 4. 
Tabla 4. Reactivos elaborados por dimensión

\begin{tabular}{lll}
\multicolumn{2}{c}{ teórica } \\
\hline \multicolumn{1}{c}{$\begin{array}{c}\text { Dimensión teórica } \\
\text { ejecución de las } \\
\text { actividad }\end{array}$} & $\begin{array}{l}\text { Emociones vinculadas con } \\
\text { los resultados obtenidos }\end{array}$ \\
\hline $\begin{array}{l}\text { Me gusta resolver los } \\
\text { ejercicios de } \\
\text { matemáticas }\end{array}$ & $\begin{array}{l}\text { Me ponen triste mis } \\
\text { calificaciones de historia }\end{array}$ \\
$\begin{array}{l}\text { Me divierto } \\
\text { escribiendo textos en } \\
\text { el cuaderno }\end{array}$ & $\begin{array}{l}\text { Me pongo feliz cuando me } \\
\text { dicen las calificaciones de } \\
\text { geografía }\end{array}$ \\
$\begin{array}{l}\text { Me gusta hacer la } \\
\text { tarea de geografía }\end{array}$ & $\begin{array}{l}\text { Estoy triste con mis } \\
\text { calificaciones de español }\end{array}$ \\
$\begin{array}{l}\text { Me gusta cuando } \\
\text { tenemos examen }\end{array}$ & $\begin{array}{l}\text { Estoy feliz con las } \\
\text { calificaciones que saco en } \\
\text { los exámenes }\end{array}$ \\
$\begin{array}{l}\text { Me gusta trabajar en } \\
\text { la clase de historia }\end{array}$ & $\begin{array}{l}\text { Me entristecen mis } \\
\text { calificaciones de } \\
\text { matemáticas }\end{array}$ \\
\hline
\end{tabular}

Tabla 5. Medias y desviaciones estándar de las puntuaciones de los jueces sobre la claridad, coherencia y relevancia de los reactivos

\begin{tabular}{|c|c|c|}
\hline Reactivo & $M$ & $D E$ \\
\hline $\begin{array}{l}\text { 1. Me gusta resolver los ejercicios de } \\
\text { matemáticas }\end{array}$ & 4 & - \\
\hline $\begin{array}{l}\text { 2. Me ponen triste mis calificaciones de } \\
\text { historia }\end{array}$ & 4 & - \\
\hline $\begin{array}{l}\text { 3. Me divierto escribiendo textos en el } \\
\text { cuaderno }\end{array}$ & 3.93 & .26 \\
\hline $\begin{array}{l}\text { 4. Me pongo feliz cuando me dicen las } \\
\text { calificaciones de geografía }\end{array}$ & 3.93 & .26 \\
\hline 5. Me gusta hacer la tarea de geografía & 3.93 & .26 \\
\hline 6. Me gusta cuando tenemos examen & 4 & - \\
\hline 7. Me gusta trabajar en la clase de historia & 4 & - \\
\hline $\begin{array}{l}\text { 8. Estoy triste con mis calificaciones de } \\
\text { español }\end{array}$ & 3.93 & .26 \\
\hline $\begin{array}{l}\text { 9. Estoy feliz con las calificaciones que } \\
\text { saco en los exámenes }\end{array}$ & 4 & - \\
\hline $\begin{array}{l}\text { 10. Me entristecen mis calificaciones de } \\
\text { matemáticas }\end{array}$ & 4 & - \\
\hline
\end{tabular}

Nota. $M=$ media aritmética; $D E=$ desviación estándar

Cabe destacar que, estos resultados permitieron explorar la variable en la muestra particular del presente estudio, lo cual favoreció la creación de ítems culturalmente relevantes para medir las emociones de logro en estudiantes de primaria de la Zona Metropolitana del Valle de México. Sin embargo, de acuerdo con los hallazgos sobre los significados, no se consideró la creación de reactivos sobre emociones de resultado prospectivas (ansiedad, esperanza, desesperanza y desesperación experimentadas durante la anticipación de la calificación obtenida), negativas (enojo, frustración, vergüenza, culpa) y de inhibición (tranquilidad, alivio, aburrimiento y decepción), porque no fueron mencionadas por los estudiantes. Las características psicométricas de la escala desarrollada se muestran en los siguientes apartados.

\section{Validez de contenido}

De acuerdo con las evaluaciones emitidas por los jueces expertos, los reactivos de la escala se consideran suficientes para medir las emociones de logro. El promedio de las puntuaciones en esta área fue de 3.8 de cuatro posibles, el cual corresponde con la opción de respuesta "Alto nivel". En este caso, la proporción de acuerdo fue adecuada $(\mathrm{RVC}=.8)$. Respecto con la claridad, coherencia y relevancia de los ítems, todos ellos mostraron valores de RVC y IVC iguales o superiores a .90 . Con base los datos y TristánLópez (2008) la escala posee evidencia de validez de contenido. En la Tabla 5 se muestran las medias de los valores otorgados por los jueces en estas dimensiones.

\section{Validez de constructo: análisis factorial exploratorio}

Una vez aplicada la Escala de Emociones de Logro para Estudiantes de Primaria a 250 estudiantes de $4^{\circ}$ a $6^{\circ}$ grado, y de acuerdo con las frecuencias y los datos obtenidos en la prueba $t$ de Student, se confirmó que; en la base de datos no existían valores fuera de rango, que todas las opciones de respuesta fueron elegidas, y que los ítems discriminaron entre los grupos extremos establecidos a partir de los percentiles 25 y 75 . Las frecuencias de elección en las opciones de respuesta oscilaron entre 8.4 y $41 \%$. Asimismo, sus valores en la $t$ de Student fueron significativos $(t \geq 5.89, \quad p<.001)$. Respecto con la asimetría, curtosis y las correlaciones ítem-total corregidas, se encontró que; las distribuciones se asemejaron a la normalidad, los valores se ubicaron dentro del rango adecuado para utilizar matriz de correlaciones de Pearson y el método de máxima verosimilitud, y que los reactivos mostraron homogeneidad (Tabla 6). Por ello, todos los ítems se incluyeron en el análisis factorial exploratorio (AFE).

El AFE mostró una estructura con dos factores que explicaron el $48.12 \%$ de la varianza. El primer factor aportó $30.79 \%$ de la explicación, y en su mayoría agrupó reactivos asociados con emociones positivas de activación, experimentadas durante la 
Tabla 6. Asimetría, curtosis y correlación ítem-total corregida de los reactivos

\begin{tabular}{lccc}
\hline Ítem & Asimetría & Curtosis & $\begin{array}{c}\text { Correlación ítem-total } \\
\text { corregida }\end{array}$ \\
\hline 1. Me gusta resolver los ejercicios de matemáticas & -.58 & -.81 & $.32^{*}$ \\
2. Me ponen triste mis calificaciones de historia & -.53 & -.38 & $.50^{*}$ \\
3. Me divierto escribiendo textos en el cuaderno & -.47 & -.79 & $.30^{*}$ \\
4. Me pongo feliz cuando me dicen las calificaciones de geografía & -.50 & -.33 & $.41^{*}$ \\
5. Me gusta hacer la tarea de geografía & -.27 & -.78 & $.32^{*}$ \\
6. Me gusta cuando tenemos examen & .30 & -.83 & $.54^{*}$ \\
7. Me gusta trabajar en la clase de historia & -.14 & -.49 & $.32^{*}$ \\
8. Estoy triste con mis calificaciones de español & -.91 & .55 & $.48^{*}$ \\
9. Estoy feliz con las calificaciones que saco en los exámenes & -.53 & -.54 & $.47^{*}$ \\
10. Me entristecen mis calificaciones de matemáticas & -.63 & -.50 & $.41^{*}$ \\
\hline
\end{tabular}

Tabla 7. Escala de Emociones de Logro para Estudiantes de Primaria: Subescalas, cargas factoriales y varianzas

\begin{tabular}{|c|c|c|c|}
\hline \multirow{2}{*}{\multicolumn{2}{|c|}{ Reactivos }} & Factor 1 & Factor2 \\
\hline & & $\begin{array}{c}\text { Emociones positivas de } \\
\text { activación durante la actividad }\end{array}$ & $\begin{array}{c}\text { Emociones retrospectivas } \\
\text { de resultado }\end{array}$ \\
\hline \multicolumn{2}{|c|}{ 5. Me gusta hacer la tarea de geografía } & .71 & 27 \\
\hline \multicolumn{2}{|c|}{ 6. Me gusta cuando tenemos examen } & .63 & .28 \\
\hline \multicolumn{2}{|c|}{ 7. Me gusta trabajar en la clase de historia } & .48 & .09 \\
\hline \multicolumn{2}{|c|}{ 3. Me divierto escribiendo textos en el cuaderno } & .48 & .19 \\
\hline \multicolumn{2}{|c|}{ 1. Me gusta resolver los ejercicios de matemáticas } & .46 & .02 \\
\hline \multicolumn{2}{|c|}{ 4. Me pongo feliz cuando me dicen las calificaciones de geografía } & .43 & .15 \\
\hline \multicolumn{2}{|c|}{ 10. Me entristecen mis calificaciones de matemáticas } & .24 & .73 \\
\hline \multicolumn{2}{|c|}{ 8. Estoy triste con mis calificaciones de español } & .02 & .71 \\
\hline \multicolumn{2}{|c|}{ 2. Me ponen triste mis calificaciones de historia } & .11 & .54 \\
\hline \multicolumn{2}{|c|}{ 9. Estoy feliz con las calificaciones que saco en los exámenes } & .20 & .44 \\
\hline \multirow[t]{2}{*}{ Número de reactivos } & Total & & \\
\hline & 10 & 6 & 4 \\
\hline$\%$ de varianza explicada & 48.12 & 30.79 & 17.33 \\
\hline
\end{tabular}

realización de las actividades escolares. El segundo mostró un aporte del $17.33 \%$ e incluyó ítems relativos con emociones retrospectivas de resultado. El valor del KMO reveló que la matriz para el cálculo del AFE fue adecuada: $\mathrm{KMO}=.75$; $\chi^{2}(45, N=250)=550.76, p<.001$. Sin embargo, el reactivo; 4. Me pongo feliz cuando me dicen las calificaciones de geografía, saturó de forma diferente a lo esperado. Teóricamente, este ítem pertenece a las emociones de resultado, pero se ubicó en las emociones experimentadas durante la realización de las actividades escolares. El resto de los reactivos se agrupó en el factor esperado de acuerdo con la clasificación de la Tabla 4, elaborada con base en las dimensiones teóricas de Frenzel et al. (2007) y en los hallazgos obtenidos sobre los significados de los estudiantes.

Con base en los datos obtenidos y los criterios establecidos por Martínez, Hernández y Hernández (2014) y López-Roldán y Fachelli (2016), fue adecuado utilizar análisis factorial para la extracción y la explicación de los datos. Todos los reactivos mostraron cargas significativas y específicas de su factor a nivel teórico. Los resultados sobre dicho análisis se muestran en la Tabla 7.

\section{Validez de constructo: análisis factorial confirmatorio}

Finalmente, con los datos de la aplicación de la escala con la muestra de 248 estudiantes de $4^{\circ}$ a $6^{\circ}$ grado de primaria, se realizó un análisis factorial confirmatorio para comprobar la estructura previa. El modelo presentó los siguientes datos e índices de ajuste: $\chi^{2}(26$, $N=248)=66.50, \quad p^{<.001}, \quad \chi^{2} / \mathrm{gl}=2.56 ; \quad$ IFI=.89, GFI $=.94, \quad$ CFI $=.89, \quad$ AGFI $=.90, \quad$ RMSEA $=.07$ y $\mathrm{RMR}=.08$. Los resultados mostraron algunos valores limítrofes y otros aceptables en los ajustes de los índices. Los datos mostraron un modelo parsimonioso, obtenido sin cambiar la estructura encontrada en el AFE, sin covarianzas entre errores y con valores superiores en todos los índices, en comparación con un modelo unifactorial, especialmente entre los ajustes comparativos. Este último presentó datos fuera de los rangos aceptables: $\chi^{2}(35, N=248)=217.87$, $p<.001, \chi^{2} / g l=6.22 ; \mathrm{IFI}=.56, \mathrm{GFI}=.83, \mathrm{CFI}=.55$, 
Tabla 8. Alfa de Cronbach, correlaciones inter-factor, media y desviación estándar de la Escala de Emociones de Logro para Estudiantes de Primaria

\begin{tabular}{lccc}
\hline & Total & $\begin{array}{c}\text { Factor 1. } \\
\text { Emociones positivas de } \\
\text { activación durante la actividad }\end{array}$ & $\begin{array}{c}\text { Factor } 2 . \\
\text { Emociones retrospectivas de } \\
\text { resultado }\end{array}$ \\
\hline $\begin{array}{l}\text { Número de reactivos } \\
\text { Alfa de Cronbach }\end{array}$ & 10 & 6 & 4 \\
Correlaciones inter-factor & $.74^{1} / .70^{2}$ & $.71^{1} / .66^{2}$ & $.70^{1} / .69^{2}$ \\
$\begin{array}{l}\text { Emociones positivas de activación } \\
\text { durante la actividad }\end{array}$ & & 1.00 & 1.00 \\
$\begin{array}{l}\text { Emociones retrospectivas de } \\
\text { resultado }\end{array}$ & & $.13, p=.04^{1}$ & $.23, p<.01^{2}$ \\
Media (media teórica =2.5) & $3.55^{1} / 3.52^{2}$ & $3.38^{1} / 3.40^{2}$ & $.78^{1} / .71^{2}$ \\
Desviación estándar & $.64^{1} / .60^{2}$ & $.65^{1} / 3.71^{2}$ \\
\hline
\end{tabular}

Nota. ${ }^{1}=$ resultado con la muestra de 250 estudiantes; ${ }^{2}=$ resultado con la muestra de 248 estudiantes.

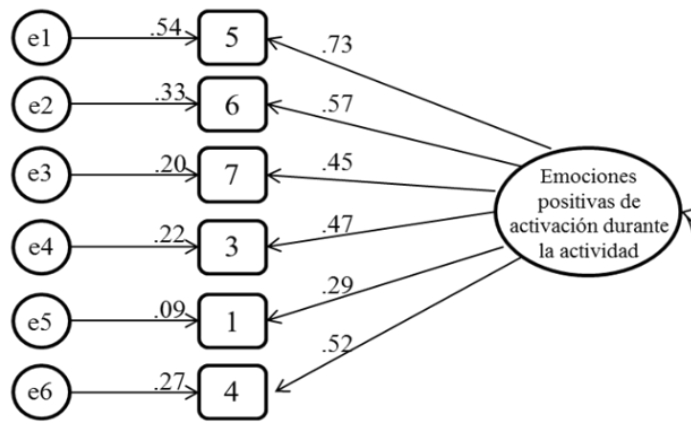

.26



Figura 1. Modelo estructural de las correlaciones de los factores de las emociones de logro

AGFI=.74, RMSEA=.14 y RMR=.154. En la Figura 1 se muestra la representación gráfica del modelo de dos factores.

En la Figura 1 se puede observar que los pesos factoriales fueron aceptables y que los reactivos; 10,8 y 2 , mostraron correlaciones negativas, puesto que, se refieren a emociones de tristeza y se encuentran en sentido inverso, en comparación con el resto de los ítems. Por el contrario, el reactivo número 9, así como los ítems del segundo factor, consideran emociones positivas, tales como felicidad, gusto y disfrute.

Debido a la distribución de los ítems y a los resultados obtenidos, la Escala de Emociones de Logro para Estudiantes de Primaria se centra en la medición de las emociones experimentadas en entornos educativos, por medio de los factores: a) emociones positivas de activación durante la actividad; y, b) emociones retrospectivas de resultado. El primero de los factores fue definido como el disfrute y la felicidad que sienten los alumnos durante la ejecución de las actividades escolares. Por su parte, el segundo se conceptualizó como la tristeza y la felicidad que sienten los estudiantes por las calificaciones obtenidas.

\section{Validez de criterio concurrente.}

Con base en las puntuaciones totales de las escalas aplicadas y en las calificaciones de la muestra de 248 estudiantes de $4^{\circ}$ a $6^{\circ}$ grado de primaria, las correlaciones de Pearson indicaron asociaciones lineales, significativas $(p<.001) \mathrm{y}$ positivas, lo que indica evidencia de validez de criterio. Con el rendimiento se obtuvo una correlación $r=.32$, y con los resultados de las sumatorias de las puntuaciones en las escalas de Motivación Escolar (Durán \& Acle, 2021), Valor a la Tarea (Durán \& Acle, 2019) y Autoconcepto Académico (Durán \& Acle, 2020), fueron de; $r=.58, r=.81$ y $r=.59$, respectivamente.

\section{Confiabilidad}

Los valores de alfa de Cronbach indicaron consistencias internas aceptables y similares en ambas sub-muestras de estudiantes. En los dos casos, todos los reactivos correlacionaron de manera significativa con el puntaje total $[r \geq .24$, $p \leq .01]$. Los resultados sobre el análisis se muestran en la Tabla 8. 


\section{Discusión}

Con base en los resultados, el objetivo del estudio fue alcanzado al construir una escala con evidencias de confiabilidad y validez en la evaluación de las emociones de logro en estudiantes de primaria. El estudio exploratorio de tipo cualitativo, permitió la identificación de las actividades de aprendizaje y las emociones vivenciadas en la escuela, información que posteriormente facilitó la elaboración de reactivos apropiados con la teoría y con las características de la muestra. En este sentido, los significados de los alumnos concordaron con la teoría de controlvalor, en que las emociones que vivencian los estudiantes en entornos escolares, surgen a partir de las actividades que realizan y por los resultados que obtienen, es decir, sus calificaciones (Pekrun et al., 2007; Pekrun et al., 2011). Por ello, la escala es coherente con la definición de emociones de logro postulada por Frenzel et al. (2007).

Además, con base en Kleine et al. (2005) y Pekrun et al. (2009) éstas pueden clasificarse con los criterios de valencia y activación, por lo que existen emociones positivas de activación, como el gusto al hacer la tarea, y las de resultado pueden considerase de manera retrospectiva, como la tristeza después de recibir las calificaciones. En este sentido, ambas formaron parte de las dimensiones identificadas en los análisis factoriales. En consecuencia, los significados de los grupos focales sirvieron para revisar la vigencia y pertinencia de este constructo en niños de nivel primaria de una cultura no estudiada anteriormente.

Sin embargo, dentro del planteamiento teórico descrito por Kleine et al. (2005) y Pekrun et al. (2009), se incluyen otras emociones, como las de resultado prospectivas (experimentadas en la anticipación de la calificación obtenida, tales como ansiedad, esperanza y desesperación), negativas (como enojo, frustración y vergüenza) y de inhibición (aburrimiento y decepción), que no fueron consideradas en la escala, porque los estudiantes no las mencionaron, lo cual, probablemente afectó la validez de contenido.

A pesar de la evidencia obtenida con los jueces sobre la suficiencia de los reactivos, parece adecuado realizar más estudios para descartar o identificar, la presencia de emociones negativas, de inhibición y de resultado prospectivas en estudiantes de primaria, y de ser necesario, complementar la Escala de Emociones de Logro para Estudiantes de Primaria con nuevos ítems.

Aunado con lo anterior, a nivel empírico se observó una discrepancia importante con los hallazgos de algunos estudios. En la investigación de Frenzel et al. (2007) se encontró que el disfrute, enojo, aburrimiento, desesperación y ansiedad, fueron las emociones más frecuentes en contextos escolares. Incluso, la ansiedad ha sido la más reportada (Pekrun et al., 2002). A diferencia de esos resultados, en el presente estudio únicamente se identificaron emociones de tristeza y disfrute, esta última con diversas nominaciones, como gusto, alegría y felicidad. Sin embargo, las discrepancias en las edades y en el desarrollo afectivo de los participantes, permiten comprender la razón de las divergencias.

La alegría y la tristeza forman parte de las emociones básicas que se experimentan en los primeros años de vida, y posteriormente surgen otras de mayor complejidad, como desesperación y ansiedad. Asimismo, la enseñanza universitaria es diferente a la enseñanza de nivel básico, especialmente en comparación con los primeros años escolares. Existen más profesores que cuentan con estilos de enseñanza heterogéneos, las actividades a realizar son diferentes, el tiempo de las clases varía entre los niveles educativos e incluso las relaciones con los docentes y pares cambian en los niveles educativos superiores. No obstante, como se mencionó anteriormente, se recomienda continuar investigando, para determinar la presencia de otras emociones en estudiantes de primaria, además del disfrute y la tristeza encontradas en este estudio.

Por otro lado, los resultados de las relaciones con otras variables corroboraron parte importante del modelo teórico control-valor. En este sentido, se concuerda con algunos estudios. Con los trabajos de Kim y Hodges (2012), Kleine et al. (2005), Pekrun et al. (2009) y Pekrun et al. (2011) respecto a la relación entre emociones de logro y motivación escolar. Con Pekrun et al. (2002) por su asociación con el valor a la actividad, y con Herrera (2014), Kleine et al. (2005), Pekrun, et al. (2009), Valle et al. (2015) y Viljaranta (2010), por su relación con el rendimiento académico. 
Además, la información de estos hallazgos sirvió como evidencia de validez de criterio de la escala desarrollada. Estas variables también han sido consideradas en algunos instrumentos para obtener evidencia de validez, por ejemplo, el Achievement Emotions Questionnaire (Pekrun et al., 2011), el AEQ-ES (Lichtenfeld et al., 2012) y el AEQ-AR (Sánchez, 2015). En el caso del TEQ de Pekrun et al. (2004) se optó por la autoeficacia, a diferencia del autoconcepto académico que fue considerado en el presente estudio, aunque son constructos estrechamente relacionados. En todos los casos, los hallazgos convergen con relaciones estadísticamente significativas entre las emociones de logro con el resto de las variables mencionadas.

Al comparar la escala obtenida con las reportadas en la literatura, es posible percatarse de algunas semejanzas y diferencias. En las similitudes se encuentran las siguientes: (a) todos los instrumentos son tipo Likert con cinco opciones de respuesta; (b) la consistencia interna obtenida en los factores es similar a las reportadas en las dimensiones del AEQ (Pekrun et al., 2011), el AEQ-ES (Lichtenfeld et al., 2012) y el AEQPA (Peixoto et al., 2015); (c) los estudios se basan en AFC como evidencia de validez de constructo. Los datos encontrados en la $X^{2} / g l$ fueron similares a los reportados por Pekrun et al. (2011), Sánchez (2015) y Peixoto et al. (2015), con valores cercanos a tres. También, los índices de ajuste mostraron valores de GFI comparables con los del AEQ (Pekrun et al., 2011), de CFI con los del AEQ-ES (Lichtenfeld et al., 2012) y de RMSEA con los datos de los AEQ (Pekrun et al., 2011), AEQ-ES (Lichtenfeld et al., 2012) y AEQ-AR (Sánchez, 2015); (d) La validez de criterio se basa en correlaciones con variables de autoesquemas, motivación escolar, valor a la tarea y rendimiento, tal como se mencionó anteriormente; (e) Los datos sobre la validez de contenido del IVC y RVC reportado en los resultados, concuerdan con el acuerdo entre jueces obtenido por Paoloni et al. (2014); y (f) el porcentaje de varianza explicada calculado en el AFE, es similar al reportado en el AEQ-AR (Sánchez, 2015).

Por su parte, se identificaron las siguientes discrepancias: (a) la cantidad de reactivos y factores. La Escala de Emociones de Logro para Estudiantes de Primaria es la que cuenta con la menor cantidad de ítems y factores. La diferencia en los casos del TEQ (Pekrun et al., 2004), AEQ (Pekrun et al., 2011) y el AEQ-AR (Sánchez, 2015) es aproximadamente de 200 ítems; (b) la cantidad de emociones y momentos en que se evalúan éstas. El instrumento de este estudio se estructura principalmente por el disfrute durante la ejecución de las actividades y la tristeza al recibir calificaciones. En comparación, todas las pruebas encontradas incluyen más de dos emociones, entre las que se encuentran; disfrute, esperanza, orgullo, alivio, enojo, vergüenza, desesperanza, ansiedad y aburrimiento, y comúnmente las miden en tres momentos diferentes; durante la clase, al estudiar y al presentar exámenes; y, (c) las muestras. La Escala de Emociones de Logro para Estudiantes de Primaria es la única que evalúa las emociones de logro en alumnos latinos de $4^{\text {to }}$ a $6^{\text {to }}$ de primaria y que comparó la estructura obtenida contra un modelo unifactorial.

Cabe señalar que sólo se encontraron dos cuestionarios para estudiantes de este nivel básico, aunque fueron creados en Alemania-EU y Portugal, el AEQ-ES de Lichtenfeld et al. (2012) y el AEQ-PA de Peixoto et al. (2015), y sólo dos fueron construidos para alumnos universitarios de habla hispana, la revisión del AEQ de Paoloni et al. (2014) y el AEQ-AR de Sánchez (2015). Otras divergencias son (a) la consistencia interna total de la escala del presente estudio, fue inferior a la del AEQ de Pekrun et al. (2011), el AEQ-ES de Lichtenfeld et al. (2012) y el AEQ-PA de Peixoto et al. (2015), (b) los índices de IFI y CFI mostraron índices limítrofes entre aceptables y no aceptables (.89), por lo que se sugiere realizar más estudios en otras muestras, para conocer su comportamiento y realizar los ajustes pertinentes, y (c) la Escala de Emociones de Logro para Estudiantes de Primaria es la única con evidencias de las tres áreas de validez. La mayoría de los cuestionarios reportados en la literatura carecen de validez de contenido, a excepción de la revisión realizada por Paoloni et al. (2014) y sólo el AEQER de Sánchez (2015) se basó en AFE y AFC para identificar validez de constructo.

Es importante mencionar que las diferencias sobre la consistencia interna total y las cantidades de reactivos, factores, emociones y momentos de evaluación, se justifican a partir de los hallazgos obtenidos en la exploración de los significados 
con los estudiantes de primaria. La decisión de incluir en los reactivos menos emociones y momentos para su evaluación, se basó en las verbalizaciones y edades de los estudiantes. La muestra del presente estudio únicamente reportó el disfrute durante la realización de las actividades y la tristeza al recibir calificaciones. Esta información redujo notablemente la cantidad de reactivos necesarios para la medición de la variable y probablemente repercutió en el alfa de Cronbach total. Sin embargo, los datos psicométricos son aceptables si se toma en cuenta que la escala sólo contiene 10 ítems. Desde esta perspectiva, sólo dos de las ocho pruebas encontradas fueron construidas para alumnos de primaria, y en comparación con el resto, son las que poseen la menor cantidad de reactivos; el AEQ-ES de Lichtenfeld et al. (2012) con 28 ítems y el AEQ-PA de Peixoto et al. (2015) con 48.

En conclusión, la prueba creada mostró ser un instrumento adecuado para obtener puntuaciones susceptibles a interpretaciones adecuadas respecto a las emociones de logro en estudiantes de primaria mexicanos. Sus características psicométricas son apropiadas y su aplicación es sencilla y rápida. Es útil en la detección de factores de riesgo y protección relacionados con el aprendizaje y rendimiento de los estudiantes. Puede ser utilizada por psicólogos y docentes en otras investigaciones relacionadas con el tema, y para instrumentar programas de intervención preventivos. No obstante, es importante señalar que se requieren análisis y estudios adicionales para establecer puntos de corte y comparaciones normativas. Asimismo, se recomienda estudiar sus características psicométricas en poblaciones similares, realizar adaptaciones adecuadas para diferentes contextos e indagar sobre la presencia de otras emociones, además de la felicidad y la tristeza reportadas en la muestra del presente estudio.

\section{Referencias}

Argibay, J. C. (2006). Técnicas psicométricas. Cuestiones de validez y confiabilidad. Subjetividad y Procesos Cognitivos, (8), 15-33. Arrivillaga, C., \& Extremera, N. (2020). Evaluación de la inteligencia emocional en la infancia y la adolescencia: Una revisión sistemática de instrumentos en castellano. Revista Iberoamericana de Diagnóstico y Evaluación - e Avaliação Psicológica, 2(55), 121-139. https://doi.org/10.21865/RIDEP55.2.09

Castillo-Navarrete, J. L., Guzmán-Castillo, A., Bustos, C., Zavala, W., \& Vicente, B. (2020). Propiedades psicométricas del Inventario SISCO-II de estrés académico. Revista Iberoamericana de Diagnóstico y Evaluación - e Avaliação Psicológica, 3(56), 101-116. https://doi.org/10.21865/RIDEP56.3.08

Creswell, J. W. (2015). Educational Research. Pearson.

Durán, T. D. D., \& Acle, G. (2019). Construcción de una Escala para Medir Valor a la Tarea en Alumnos de Primaria. Actas del XIX Congreso Internacional de Investigación Educativa (Vol. II). https://aidipe2019.aidipe.org/files/2019/06/Ac tas_AIDIPE2019_Vol_II.pdf

Durán, T., \& Acle, G. (2020). Evidencias de validez y confiabilidad de una escala para evaluar autoconcepto académico. Psicología Iberoamericana, 28(2).

https://psicologiaiberoamericana.ibero.mx/ind ex.php/psicologia/article/view/320/569

Durán, T. D. D., \& Acle, G. (2021). Escala de Motivación Escolar para Alumnos de Primaria: Evidencias de validez $y$ confiabilidad. En prensa.

Escobar-Pérez, J., \& Cuervo-Martínez, A. (2008). Validez de contenido y juicio de expertos: Una aproximación a su utilización. Avances en Medición, 6, 27-36.

http://www.humanas.unal.edu.co/psicometria/ files/7113/8574/5708/Articulo3_Juicio_de_ex pertos_27-36.pdf

Fernández, R. (2008). Modelos de medida y análisis factorial confirmatorio. En M. A. Verdugo, M. Crespo, M. Badía, y B. Arias (Coords.), Metodología en la investigación sobre discapacidad. Introducción al uso de las ecuaciones estructurales (pp. 29-41). Publicaciones del INICO. http://riberdis.cedd.net/bitstream/handle/1118 1/3270/Metodologia_en_investigacion_sobre_ discapacidad.pdf? sequence $=2 \&$ rd $=003125214$ 5808643 
Ferrando, P. J., \& Lorenzo-Seva, U. (2014). El análisis factorial exploratorio de los ítems: Algunas consideraciones adicionales. Anales de Psicología, 30(3), 1170-1175. http://dx.doi.org/10.6018/analesps.30.3.199991

Frenzel, A. C., Pekrun, R., \& Goetz, T. (2007). Perceived learning environment and students' emotional experiences: A multilevel analysis of mathematics classrooms. Learning and Instruction, 17(5), 478-493. doi:10.1016/j.learninstruc.2007.09.001

Herrera, F. (2014). Motivación, estrategias de aprendizaje y rendimiento académico, en alumnos de Nutrición y Dietética de la Universidad del Desarrollo. Revista Educación, Ciencia y Salud, 11(1), 38-46. http://www2.udec.cl/ofem/recs/anteriores/vol1 112014/artinv11114e.pdf

Jiménez-Blanco, A., Sastre, S., Artola, T., \& Alvarado, J. M. (2020). Inteligencia emocional y rendimiento académico: Un modelo evolutivo. Revista Iberoamericana de Diagnóstico y Evaluación - e Avaliação Psicológica, 3(56), 129-141. https://doi.org/10.21865/RIDEP56.3.10

Kerlinger, F., \& Lee, H. B. (2002). Investigación del comportamiento. Métodos de investigación en ciencias sociales. McGrawHill.

Kim, C., \& Hodges, C. B. (2012). Effects of an emotion control treatment on academic emotions, motivation and achievement in an online mathematics course. Intr Sci, 40, 173192.

https://www.academia.edu/2448621/Effects_o f_an_emotion_control_treatment_on_academi c_emotions_motivation_and_achievement_in _an_online_mathematics_course

Kleine, M., Goetz, T., Pekrun, R., \& Hall, N. (2005). The structure of students' emotions experienced during a mathematical achievement test. Analyses, 37(3), 221-225. doi:10.1007/s11858-005-0012-6

Lichtenfeld, S., Pekrun, R., Stupnisky, R. H., Reiss, K., \& Murayama, K. (2012). Measuring students' emotions in the early years: The Achievement Emotions QuestionnaireElementary School (AEQ-ES). Learning and individual differences, 22(2), 190-201. doi:10.1016/j.lindif.2011.04.009
Lloret-Segura, S., Ferreres-Traver, A., HernándezBaeza, A., \& Tomás-Marco, I. (2014). El análisis factorial exploratorio de los ítems: Una guía práctica, revisada y actualizada. Anales de Psicología, 30(3), 1151-1169. http://dx.doi.org/10.6018/analesps.30.3.199361

López-Roldán, P., Fachelli, S. (2016). Análisis factorial. En P. López-Roldán, \& S. Fachelli, Metodología de la Investigación Social Cuantitativa. Bellaterra (Cerdanyola del Vallès): Dipòsit Digital de Documents, Universitat Autònoma de Barcelona. $1^{\text {a }}$ edición, versión 3. Edición digital: http://ddd.uab.cat/record/142928

Martínez, M. R., Hernández, M. J., \& Hernández, M. V. (2014). Psicometría. Alianza Editorial.

Montero, I., \& León, O. G. (2007). A guide for naming research studies in Psychology. International Journal of clinical and Health psychology, 7(3), 847-862.

Paoloni, P. V., Vaja, A. B., \& Muñoz, V. L. (2014). Reliability and validity of the Achievement Emotions Questionnaire. A study of Argentinean University students. Electronic Journal of Research in Educational Psychology, 12(3), 671-692. doi:10.14204/ejrep.34.14088

Peixoto, F., Mata, L., Monteiro, V., Sanches, C., \& Pekrun, R. (2015). The achievement emotions questionnaire: Validation for preadolescent students. European Journal of Developmental Psychology, 12(4), 472-481. doi:10.1080/17405629.2015.1040757

Pekrun, R., Elliot, A. J., \& Maier, M. A. (2009). Achievement goals and achievement emotions: Testing a Model of their joint relations with academic performance. Journal of Education Psychology, 101(1), 115-135. http://citeseerx.ist.psu.edu/viewdoc/download ?doi=10.1.1.448.9758\&rep=rep1\&type=pdf

Pekrun, R., Frenzel, A. C., Goetz, T., \& Perry, R. P. (2007). The control-value theory of achievement emotions: An integrative approach to emotions in educations. Emotion in education, 13-36.

https://psycnet.apa.org/doi/10.1016/B978012372545-5/50003-4

Pekrun, R., Goetz, T., Frenzel, A. C., Barchfeld, P., \& Perry, R. P. (2011). Measuring emotions in students' learning and performance: The 
Achievement Emotions Questionnaire (AEQ). Contemporary Educational Psychology, 36(1), 36-48.

http://nbn-resolving.de/urn:nbn:de:bsz:352137324

Pekrun, R., Goetz, T., Perry, R. P., Kramer, K., Hochstadt, M., \& Molfenter, S. (2004). Beyond test anxiety: Development and validation of the Test Emotions Questionnaire (TEQ). Anxiety, Stress \& Coping, 17(3), 287316.

https://doi.org/10.1080/106158004123313038 47

Pekrun, R., Goetz, T., Titz, W., \& Perry, R. P. (2002). Academic emotions in students' selfregulated learning and achievement: A program of qualitative and quantitative research. Educational Psychologist, 37(2), 91106. https://d-nb.info/1115472046/34

Pérez, J. A., Chacón, S., \& Moreno, R. (2000). Validez de constructo: El uso de análisis factorial exploratorio-confirmatorio para obtener evidencias de validez. Psicothema, 12(Su2), 442-446.

Pintrich, P. R., \& Schunk, D. H. (2006). Motivación en contextos educativos: Teoría, investigación y aplicaciones. Pearson educación.

Rea, A. C., \& Acle, G. (2017). Situación de vulnerabilidad y estrategias resilientes de un grupo de padres de hijos con trastorno del espectro autista (TEA). Revista COCAR, 11(21), 8-18.

https://pdfs.semanticscholar.org/249d/527338 30f6f45864a93e1c40a801b31141be.pdf

Reyes-Lagunes, I., García, \& Barragán, L. F. (2008). Procedimiento de validación psicométrica culturalmente relevante: Un ejemplo. La Psicología Social en México, 12, 625-636.

https://www.researchgate.net/publication/259 191173

Salvo, L., \& Castro, A. (2013). Confiabilidad y validez de la escala de impulsividad de Barratt (BIS-11) en adolescentes. Revista Chilena de Neuro-Psiquiatría, 51(4), 245-254.

https://scielo.conicyt.cl/pdf/rchnp/v51n4/art03 .pdf

Sánchez, J. (2015). The Achievement Emotions Questionnaire-Argentine (AEQ-AR): Internal and external validity, reliability, gender differences and norm-referenced interpretation of test scores. Evaluar, 15(1), 41-74.

https://www.researchgate.net/publication/305 680338

Santos-Roig, M., \& Pérez-Meléndez, C. (2014). Análisis de ítems y evidencia de fiabilidad de la Escala sobre Representación Cognitiva de la Enfermedad (ERCE). Anales de Psicología, 30(2), 438-449.

http://dx.doi.org/10.6018/analesps.30.2.164021

Tristán-López, A. (2008). Modificación al modelo de Lawshe para el dictamen cuantitativo de la validez de contenido de un instrumento objetivo. Avances en Medición, 6, 37-48. https://pdfs.semanticscholar.org/4017/81bbf69 1f0c15965fcf667e98a48e4899165.pdf

Valle, A., Regueiro, B., Estévez, I., Piñeiro, I., Rodríguez, S., \& Freire, C. (2015). Implicación y motivación hacia los deberes escolares en los estudiantes de Primaria según el rendimiento académico y el curso. European Journal of Investigation in Health, 5(3), 345-355.

https://www.researchgate.net/publication/297 507985_Implicacion_y_motivacion_hacia_los _deberes_escolares_en_los_estudiantes_de_Pr imaria_segun_el_rendimiento_academico_y_e 1_curso

Viljaranta, J. (2010). The development and role of task motivation and task values during different phases of the school career. Jyväskylä Studies in Education, Psychology and Social Research, (401).

Weiner, B. (1972). Attribution Theory, achievement motivation, and the educational process. Review of Educational Research, 42(2), 203-215.

https://www.researchgate.net/publication/270 334287_Attribution_Theory_Achievement_M otivation_and_the_Educational_Process

Weiner, B. (1985). An attributional theory of achievement motivation and emotion. Psychological Review, 92(4), 548-573. http://acmd615.pbworks.com/f/weinerAnattrib utionaltheory.pdf

Weiner, B. (2010). Attribution theory. International Encyclopedia of Education, 6, 558-563. 
doi:10.1002/9780470479216.corpsy0098

Wigfield, A., \& Eccles, J. S. (1992). The development of achievement task values: A theoretical analysis. Developmental Review, $12,265-310$. doi:10.1016/0273-2297(92)90011-P

Wigfield, A., \& Eccles, J. S. (2000). ExpectancyValue Theory of Achievement Motivation. Contemporary Educational Psychology. 25, 68-81.

https://pdfs.semanticscholar.org/0a28/c12a021 40983603c7231ebae70564066f86b.pdf 